\title{
Structure and dynamics of the Milky Way disk as revealed from the radial velocity distributions of APOGEE red clump stars
}

\author{
Daisuke Toyouchi and Masashi Chiba \\ Astronomical Institute, Tohoku University, Aoba-ku, Sendai 980-8578, Japan \\ email: toyouchi@astr.tohoku.ac.jp \\ email: chiba@astr.tohoku.ac.jp
}

\begin{abstract}
We investigate the structure and dynamics of the Milky Way (MW) disk stars based on the analysis of the Apache Point Observatory Galactic Evolution Experiment (APOGEE) data, to infer the past evolution histories of the MW disk component(s) possibly affected by radial migration and/or satellite accretions. APOGEE is the first near-infrared spectroscopic survey for a large number of the MW disk stars, providing their radial velocities and chemical abundances without significant dust extinction effects. We here adopt red-clump (RC) stars (Bovy et al. 2014), for which the distances from the Sun are determined precisely, and analyze their radial velocities and chemical abundances in the MW disk regions covering from the Galactocentric distance, $R$, of $5 \mathrm{kpc}$ to $14 \mathrm{kpc}$. We investigate their dynamical properties, such as mean rotational velocities, $\left\langle V_{\phi}\right\rangle$ and velocity dispersions, as a function of $R$, based on the MCMC Bayesian method. We find that at all radii, the dynamics of alpha-poor stars, which are candidates of young disk stars, is much different from that of alpha-rich stars, which are candidates of old disk stars. We find that our Jeans analysis for our sample stars reveals characteristic spatial and dynamical properties of the MW disk, which are generally in agreement with the recent independent work by Bovy et al. (2015) but with a different method from ours.
\end{abstract}

Keywords. Galaxy: disk,Galaxy: evolution, Galaxy: kinematics and dynamics, Galaxy: structure

\section{Results and Discussion}

The results in this work can be understood with the asymmetric drift equation, which shows that the form of $\left\langle V_{\phi}\right\rangle(R)$ depends on the radial profile of velocity dispersion and stellar density. One of the remarkable results is that although the radial profiles of velocity dispersions for alpha-poor populations are nearly flat, their $\left\langle V_{\phi}\right\rangle(R)$ rapidly declines at $\mathrm{R} \sim 9 \mathrm{kpc}$. The asymmetric drift equation then suggests that the gradient of stellar density for alpha-poor populations around this radius also rapidly declines. On the other hand, the flat radial profile of $\left\langle V_{\phi}\right\rangle(R)$ for alpha-rich population implies that the radial gradient of their stellar density is nearly constant. Such radial density structures of the Galactic stellar disk implied here are generally consistent with the recent work based on the statistical analysis in the spatial distribution of APOGEE-RC stars by Bovy et al. (2015). More detailed discussion for our results will be presented in Toyouchi \& Chiba (2016) in preparation.

\section{References}

Bovy, J., Nidever, D. L., Rix, H.-W., et al. 2014, ApJ, 790, 127

Bovy, J., Rix, H.-W., Schlafly, E. F., et al. 2016, ApJ, 823, 30 